Aus dem k. k. großen mobilen Epidemielaboratorium 5 und einem Epidemiespital des IV. A.-K.

\section{Die zerebralen Erscheinungen und die meningeale Permeabilität bei Fleckfieber.}

\section{Von E. Weil und A. Soucek. \\ I. Klinischer Teil (Soucek).}

Die Vielgestaltigkeit, unter der das Fleckfieber auftritt, haben in diesem Kriege viele Aerzte oft mit Schmerzen sich eingestehen müssen. Dennoch haftet ein gemeinsamer Zug den mannigfachen Bildern dieser Krankheit an, und das sind meningitische und damit zusammenhängende zerebrale Symptome. Mit großer Regelmäßigkeit finden wir Nackensteifigkeit und Kernigsches Phänomen. Bei Prüfung dieser Erscheinung wird man, wie es z. B. bei der Meningitis cerebrospinalis oft der Fall ist, auf stärkere oder geringere Grade von Nackensteifigkeit oder Starrheit der Beugemuskeln der Ober- und Unterschenkel stoßen. Häufig nehmen die Kranken schon eine eigentümliche Haltung ein, die uns die erwähnten Symptome vermuten läßt, sie liegen mit nach hinten gebogenem Kopfe und an den Leib gezogenen Beinen im Bette.

Weniger konstant, aber doch sehr häufig findet sich der eingezogene Bauch (Kahnbauch), Dermographismus, Fußklonus. Bei vielen Kranken schwindet das Bewußtsein, sie knirschen dann oft mit den Zähnen (klonischer Krampf der Kaumuskulatur), der Blick ist starr, der Lidschlag selten. Bei manchen unterbricht diesen Zustand der Starrheit ein Delirium. Häufig zeigen die Hände einen eigentümlichen Tremor, wie er sich auch mitunter bei schwer meningitiskranken Kindern im späteren Stadium der Krankheit findet.

Der Puls ist meiner Erfahrung nach meist mehr oder minder beschleunigt, selten während des Fiebers verlangsamt.

Ebenso wie bei der Meningitis kommt es auch beim Fleckfieberkranken zu einer Neuritis optica (Arnoldsche Neuritis), zur Schwerhörigkeit und Taubheit (Neuritis nervi acustici), zu oft ganz unverhältnismäßig starker Abmagerung (trophische Störungen). Hierher gehört auch die Temperatursteigerung noch wochenlang nach der Entfieberung.

Als ein Beispiel für das meningitische Bild des Fleckfiebers sei eine Krankengeschichte mitgeteilt.

Prot. Nr. 535. Eias Sü., 12 Jahre alt, erkrankte angeblich am 24. Februar 1917 mit heftigen Kopfschmerzen, seit einigen Tagen bemerken die Angehörigen auch Schwerhörigkeit an ihm.

Befund bei der Aufnahme am 3. März: Nicht benommen, hört schwer, klagt über Kopfschmerzen, Konjunktiven leicht gerötet, Zunge dick graubraun belegt, Zungen rand frei, Rachen gerötet, leich te Schuppung der Haut des Gesichtes, des Stammes und der Extremitäten. Am Stamm, weniger dicht an den Extremitäten, ein kleinfleckiges, hirse- bis hanfkorngrc Bes, verwaschenes, hellbraunes Exanthem, Pupillen ungleich, prompt reagierend, mäßige Nackensteifigkeit, Lungen frei, Abdomen eingezogen, Milz vergrößert, deutliches Kernigsches Phänomen, Patellar. reflexe lebhaft, Dermographismus, Temperatur 38,3 , Puls 92 . Bei der Lumbalpunkticn entleert sich klarer Liquor unter erhöhtem Druck. Weil-Felix $1: 2000$ positiv.

5. März. Kopf frei, ohne Schmerzen, beweglich, kein Kopfschmerz, geringes Ohrensausen, Pupillen prompt reagierend, ungleich weit. Exanthem abgeblaßt, Zunge belagfrei, Bauch eingezogen, Milz tastbar, Kernig weniger deutlich, Patellarsehnenreflexe lebhaft, Fußklonus, Dermographismus, feinlamellöse Hautschuppung.

6. März. Entfiebert, Temperatur 36,5, Puls 60. Wohlbefinden.

7. März. Erbrechen, sonst Wohlbefinden. Weil-Felix 1:5000 positiv.

8. März. Reflexe lebhaft, Kernigsches Phänomen noch angedeutet, Banch eingezogen, Abmagerung.

10. März. Weil-Felix 1:2000 positiv. Die Lumbalpunktion ergibt klaren Liquor unter nicht erhöhtem Druck.

12. März. Pupillen ungleich, Ohrensausen, Kernigsches Phänomen angedeutet, Bauchdecken- und Patellarsehnenreflexe lebhaft, Fußklonus, Temperatur 36,8 , Puls 72 , mehrere schleimige Stühle, bakteriologisch Dysenterie Flexner.

15. März.: Lidtremor, Pupillendifferenz, Temperatur 36, Puls 84 Wohlbefinden

Wir hatten es im beschriebenen Fall mit einem Flecktyphus zu tun, der verhältnismäßig leicht verlief, dennoch aber die meningealen Symptome deutlich aufwies.
Die meningealen und die damit zusammenhängenden zerebralen Erscheinungen, die wir mehr oder weniger mit einer gewissen Regelmäßigkeit stets bei Fleckfieber finden, könnten nun entweder ohne nachweisbare anatomische Veränderungen der Meningen sein, wie beim Meningismus, bei der Spitzenpneumonie, oder es können auch nachweisbare Veränderungen der Meningen zugrunde liegen.

Mit diesem Nachweis beschäftigt sich der zweite Teil dieser Arbeit.

\section{Liquoruntersuchungen (W eil).}

Im Vorangehenden wurde auf die Notwendigkeit hingewiesen, für die klinischen Erscheinungen von seiten des Zentralnervensystems eine nachweisbare Grundlage zu finden. Wie man aus der Beschaffenheit des Harnes auf die Erkrankungen der Niere schließen kann, so weist uns die Lumbalflüssigkeit auf bestimmte Affektionen des zentralen Nervensystems hin. Am eingehendsten sind nach dieser Richtung die meningitischen Veränderungen studiert, die sich aus dem Lumbalpunktat mit großer Wahrscheinlichkeit erkennen lassen. Schon seit langer Zeit galt die Eiweiß- und Zellvermehrung im Liquor als diagnostischer Behelf für die entzündlichen meningealen Erkrankungen, und erst in den letzten Jahren wurde durch den Nachweis der erhöhten Permeabilität der Meningen für Immunkörper (Hämolysinreaktion von Weil und Kafka) nicht nur in theoretischer, sondern auch praktischer Hinsicht ein Gewinn erzielt. Denn es hatte sich im Verlaufe dieser Untersuchungen gezeigt, daß die erhöhte Durchlässigkeit der Meningen völlig unabhängig vom Eiweißgehalt des Liquors auftritt, daß sie bei stärkster Eiweißvermehrung fehlen und bei normalem Eiweißgehalt in starkem Maße vorhanden sein kann. Die gegenteilige Behauptung v. $\mathrm{n}$ Za loc iecki ist allseits widerlegt worden. Es konnte festgestellt werden, daß ausschließlich ${ }^{1}$ ) bei meningitischen Prozessen akuter Natur und bei der progressiven Paralyse eine erhöhte Durchlässigkeit für die Normalhämolysine des Menschenserums besteht und daß durch die Hämolysinreaktion mit Sicherheit eine Differentialdiagnose zwischen Meningismus, Meningitis und Hirnabszeß, sowie zwischen progressiver Paralyse, chronischer Lues cerebri und unkomplizierter Tabes zu stellen ist. Diese Fragen wurden an einem großen Material von Verfasser und Schleissner bearbeitet und bereits vor dem Kriege abgeschlossen, konnten aber bisher nicht veröffentlicht werden. G. Salus hat sich auf Grund seiner Untersuchungen über den diagnostischen Wert der Hämolysinreaktion sehr günstig geäußert. Es ist sehr zu bedauern, daß bei der überaus großen Zahl der Liquoruntersuchungen im Frühıstadium der Lues die Hämolysinreaktion keine Berücksichtigung fand, denn nur durch sie allein ist hier eine Ent. scheidung zu treffen; man kann schon heute feststellen, daß die übrigen Reaktionen mit Ausnahme der Wassermannschen zur Lösung der dabei angestrebten Fragen ziemlich wertlos sind.

Da bei der Hämolysinreaktion der Uebertritt der normalerweise im Blute vorhandenen Antikörper nachgewiesen wird, so ist es klar, daß die erhöhte Permeabilität auch den Nachweis der bei Infektionskrankheiten im Blute auftretenden Immunkörper gestatten mïisse.

Durch die Fleckfieberagglutination war uns die Möglichkeit geboten, im Liquor nach den Agglutininen zu suchen. D. F uchs war der Erste, welcher über diesbezügliche Befunde berichtete. $\left.{ }^{2}\right)$ Er fand bei acht Fällen viermal Agglutination im Liquor, sogar gegen den schwer agglutinablen Stamm $X_{2}$, und mißt diesem Resultate eine große Bedeutung bei. Nun sind schon bei einer ganzen Reihe von Infektionskrankheiten Liquoruntersuchungen vorgenommen worden. Diese haben ziemlich übereinstimmend das Resultat ergeben, daß nur bei Meningitiden und lokalen Prozessen im Zentralnervensystem Antikörper in nachweisbarer Menge in der Lumbalflüssigkeit vorhanden sind. So hat man bei Meningitis epidemica und tuberculosa komplementbindende Antistoffe und Agglutinine, bei Lues cerebri, Tabes und Paralyse positiven Wassermann nachgewiesen. Es kommt

1) Eine erhöhte Durchlässigkeit tritt auch bei jenen seltenen Tumoren des Zentralnervensystems auf, bei welchen es zu einer Unterbrechung der Liquorzirkulation kommt, doch hat sie hier selbstverständlich eine ganz andere Ursache.

$\left.{ }^{2}\right)$ Feldärztl. Blätter d. II. A.-K. Nr. 16. 
jedoch darauf an festzustellen, ob die im Liquor auftretenden Antikörper lokal entstanden, oder ob sie infolge der erhöhten Durchlässigkeit der Meningealgefäße aus dem Blute übergetreten sind. Diese Entscheidung ist leicht und einwandfrei zu treffen, da im ersteren Falle keine bedeutende Differenz zwischen Antikörpermenge im Blute und Liquor besteht, im letzteren Falle diese sehr groß ist. Nun ist aber die normalerweise bestehende Permeabilität der Meningen zu ermitteln, welche den Uebertritt der Immunkörper des Blutes in die Lumbalflüssigkeit regelt. Gelegentlich der Untersuchungen über die Hämolysinreaktion konnten wir feststellen, daß in $70 \mathrm{ccm}$ normalem Liquor nicht soviel Hammelblutambozeptoren vorhanden sind, um $1 \mathrm{ccm}$ einer $5 \%$ igen Blutaufschwemmung zu sensibilisieren. Da $0,05 \mathrm{ccm}$ normalen Menschenserums dies vermögen, so ist das normalerweise bestehende Verhältnis von $1: 1000$ sicher nicht zu niedrig berechnet.

Bezüglich der bakteriellen Antikörper, die ja bei unseren jetzigen Versuchen als Vergleich in Betracht kommen, liegen bereits Angaben hinsichtlich des Typhus abdominalis vor. Die Befunde fast sämtlicher Autoren, Widal und Sicard, Sicard, Salo mon, Ciuca ${ }^{1}$ ), stimmen darüber überein, daß die Typhusagglutinine im Liquor nicht nachweisbar sind, auch wenn der Bluttiter sehr hoch ist. Zalociecki, der diese Frage einer genauen Untersuchung unterzog, fand das Verhältnis zwischen Agglutininen des Blutes und Liquors bei Typhus wie $1: 800$ bis 2000. Unsere eigenen Resultate an Typhen und Paratyphen, die uns als ein geeignetes Versuchsmaterial für die Fleckfieberuntersuchungen schienen, sind folgende:

Die Entnahmen wurden am Beginne der Deferveszenz, jedoch noch zur Zeit des hohen Fiebers, vorgenommen.

Fall Z. Typhus abdominalis. Agglutination im Blut $1: 500$. Agglutination im Liquor $1 \mathrm{ccm}$ negativ. Permeabilitätsverhältnis mindestens $1: 1000$.

Fall Skr. Paratyphus B. Agglutination im Blut 1:500. Paratyphus B $1: 1000$. Agglutination im Liquor Ty $1 \mathrm{ccm}$ negativ, B $1 \mathrm{ccm}$ schwach positiv. Permeabilitätsverhältnis B $1: 1000$.

Fall H. Typhus abdominalis. Agglutination im Blut $1: 5000$. Agglutination im Liquor $1 \mathrm{ccm}$ negativ. Permeabilitätsverhältnis höher als $1: 5000$.

Fall Kom. Paratyphus B. Agglutination im Blut B $1: 1000$. Agglutination im Liquor $1 \mathrm{ccm}$ negativ. Permeabilitätsverhältnis höher als $1: 1000$.

Fall Bi. Paratyphus B. Agglutination im Blut B $1: 2000$. Agglutination im Liquor $1 \mathrm{ccm}$ negativ. Permeabilitätsverhältnis höher als $1: 2000$.

Fall Gm. Paratyphus B. Agglutination im Blut B 1:500. Agglutination im Liquor $1 \mathrm{ccm}$ negativ. Permeabilitätsverhältnis mindestens $1: 1000$.

Fall Lu. Paratyplus A. Agglutination im Blut Ty $1: 10000$, A $1: 10000$. Agglutination im Liquor Ty 0,5 positiv, A $1 \mathrm{ccm}$ positiv. Permeabilitätsverhältnis für Ty $1: 5000$, für A $1: 10000$.

Fall No. Typhus abdominalis. Agglutination im Blut $1: 500$. Agglutination im Liquor $1 \mathrm{ccm}$ negativ. Permeabilitätsverhältnis min. destens $1: 1000$.

Von den 8 untersuchten Fällen besteht nur bei einem das Permeabilitätsverhältnis von $1: 1000$, bei 3 , die nur einen Blu ttiter von $1: 500$ aufweisen, läßt sich nicht sagen, $u b$ es nicht höher liegt, sicher höher jedoch ist es bei den übrigen 4 Fällen. Es läßt sich auf Grund dieser Feststellung, die in Uebereinstimmung mit den Ermittelungen früherer Autoren steht, mit Bestimmtheit behaupten, daß ein Antikörperverhältnis zwischen Blut und Liquor, das unter 1:1000 liegt, auf eine erhöhte Durchlässigkeit der Meningealgefäße hinweist.

Fin ganz anderes Bild geben jedoch, wie die nachfolgende Zusammenstellung zeigt, die Fleckfieberfälle.

Fall N. H. 12. Krankheitstag. Agglutination im Blut $\mathrm{X}_{2} 1: 200$, $X_{19} 1: 2000$. Agglutination im Liquor $X_{2} 1 \mathrm{ccm}, X_{19} 0,1$. Permeabilitätsverhältnis für $X_{2}$ und $X_{19} 1: 200$.

Nach 6 Tagen: Agglutination im Blut $X_{19} 1: 2000$. Agglutination im Liquor 0,1 . Permeabilitätsverhältnis $1: 200$.

Fall N. W. 12. Krankheitstag. Agglutination im Blut $X_{2} 1: 200$, $X_{10} 1: 5000$. Ty 500. Agglutination im Liquor $X_{2} 0,25, X_{12} 0,01$. Ty 0,25. Permeabilitätsverhältnis für $X_{2}$ und $X_{19} 1: 50$. Für Ty 1:100. Eiweiß zweifach vermehrt.

1) zit. nach Zalociecki, Arch. f. Hyg. 80 .
Nach 6 Tagen: Agglutination im Blut $X_{2} 1: 200, X_{1} 1: 2000$. Ty $1: 500$. Agglutination im Liquor $X_{2} 0,5, X_{19} 0,05$. Ty 0,25 . Permeabilitätsverhältnis für $X_{2}, X_{19}$ und Ty $1: 100$.

Fall Schi. 11. Krankheitstag. Agglutination im Blut $X_{19} 1: 1000$. Agglutination im Liquor 0,1. Permeabilitätsverhältnis $1: 100$.

Nach 2 Tagen: Agglutination im Blut $X_{19} 1: 2000$, Ty $1: 200$, B 1 : 500. Agglutination im Liquor $X_{1}, 0,05$, Ty $1 \mathrm{ccm}, \mathrm{B} 0,25$. Permeabilitätsverhältnis $X_{1}, 1: 100$, Ty $1: 200$, B $1: 100$.

Nach 14 Tagen: Agglutination im Blut $\mathrm{X}_{19} 1: 2000$, Ty $1: 200$, B $1: 200$. Agglutination im Liquor $X_{10}, 0,5$. Permeabilitätsverhältnis für $X_{19} 1: 1000$.

Fall Schei. 7. Krankheitstag. Agglutination im Blut $X_{10} 1: 200$. Agglutination im Liquor $1 \mathrm{ccm}$ negativ.

Nach 5 Tagen: 12. Krankheitstag. Agglutination im Blut X19 $1: 500$. Agglutination im Liquor 0,25. Permeabilitätsverhästnis $1: 100$.

Fall Ca. 12. Krankheitstag. Agglutination im Blut $X_{10} 1: 5000$. Agglutination im Liquor 0,01. Permeabilitätsverhältnis $1: 50$.

Fall Kr. 4. Tag nach der Entfieberung. Agglutination im Blut $X_{19} 1: 5000$. Agglutination im Liquor 0,1. Permeabilitätsverhältnis $1: 500$.

Diese Fälle zeigen ausnahmslos eine Erhöhung der Permeabilität, die erst im Verlaufe der Erkrankung auftritt und nach Ablauf derselben mehr oder weniger rasch schwindet, wie es bei heilenden Meningitiden der Fall ist.

Soweit waren unsere Untersuchungen gediehen, als wir von Felix (Konstantinopel) und Starkenstein (Radom) die Nachricht erhielten, daß bei Fleckfieber nahezu ausnahmslos in einem gewissen Stadium der Krankheit eine positive Hämolysinreaktion im Liquor zu konstatieren ist, und Starkenstein gründet darauf die Therapie des Fleckfiebers. Die beiden genannten Arbeiten sind unabhängig und unbeeinflußt von unseren Untersuchungen entstanden. Da wir inzwischen über Meerschweinchenserum und Hammelblut verfügten, konṇten wir uns an einer Anzahl von Fällen von der Richtigkeit der Angaben von Felix und Starkenstein überzeugen.

Fall Sü. $\left.{ }^{1}\right)$ 9. Krankheitstag. Agglutination im Blut $X_{2} 1: 200$, $X_{19} 1: 2000$. Agglutination im Liquor $X_{2} 0,25, X_{19} 0,05$. Permeabilitätsverhältnis für $X_{2} 1: 50$, für $X_{19} 1: 100$. Diweiß und Zellen normal. Hämolysinreaktion ohne Komplement geringe Lösung, mit Komple. ment +++ .

Nach 9 Tagen: Agglutination im Blut $X_{2} 1: 500, X_{19} 1: 2000$. Agglutination im Liquor $X_{2} 1 \mathrm{ccm}$ negativ, $X_{10} 0,5$. Permeabilitäts. verhältnis für $X_{1 g} 1: 1000$. Hämolysinreaktion negativ.

Fall Sko. 12. Krankheitstag. Agglutination im Blut $\mathrm{X}_{19} 1: 2000$. Agglutination im Liquor 0,1. Permeabilitätsverhältnis $1: 200$. Eiweiß wenig vermehrt, Zellen stark vermehrt. Hämolysinreaktion mit Kom. plement $\left.t^{2}\right)++$

Fall Schü. Agglutination im Blut $X_{2} 1: 200, X_{10} 1: 10000$. Agglutination im Liquor $X_{2} 1 \mathrm{ccm}, X_{1,} 0,025$. Permeabilitätsverhältnis für $X_{2} 1: 200$, für $X_{19} 1: 250$. Eiweiß und Zellen normal. Hämolysinreaktion mit Komplement ++ .

Fall Ko. 14. Krankheitstag. Agglutination im Blut $X_{2} 1: 200$, $X_{19} 1: 2000$. Agglutination im Liquor $X_{2} 0,5, X_{19} 0,05$. Permeabilitätsverhältnis für $X_{2}$ und $X_{1}, 1: 100$. Eiweiß schwach, Zellen stark vermehrt. Hämolysinreaktion mit Komplement +++ .

Fall $Z_{1}$. 11. Krankheitstag. Agglutination im Blut $X_{19} 1: 1000$. Agglutination im Liquor 0,1. Permeabilitätsverhältnis $1: 100$. Eiweiß und Zellen normal, Hämolysinreaktion mit Komplement ++ . Hämolyse im Blut: 0,025 Serum haben $0,5 \mathrm{ccm} 5 \%$ Hammelblut komplett gelöst.

Fall $Z_{2}$. 11. Krankheitstag. Agglutination im Blut $X_{10} 1: 200$. Agglutination im Liquor 0,5. Permeabilitätsverhältnis $1: 100$. Ejweiß und Zellen normal. Hämolysinreaktion negativ. Hämolyse im Blut: 0,05 Serum haben $0,5 \mathrm{ccm} 5 \%$ Hammelblut mäßig gelöst.

Aus den hier mitgeteilten Fällen geht hervor, daß bei 5 von $6 \mathrm{Kranken}$ die Hämolysinreaktion positiv ist. Das einzige negative Resultat dürfte auf die verringerte Ambozeptorenmenge im Blute zurückzuführen sein. Bezüglich der Stärke der Durchlässigkeit zeigen die hier untersuchten 12 Fälle bei der Annahme des normalerweise bestehenden Permeabilitätsverhältnisses von $1: 1000$ Folgendes: Bei Fall $\mathrm{Kr}$. findet sich eine doppelte; bei den Fällen N. H., Schü. und Sko. eine fünffache, bei den Fällen Schi., Schei., $Z_{1}, Z_{2}, K_{0}$. eine zehnfache,

1) Fall Elias Sü. des ersten Teiles

2) Die 5 letzten Fulle wurden von auswärts eingessandt, sodaß $\mathrm{der}$ Komplementmangel damit zusammenhängen kann. 
und bei den Fällen N. W., Ga. und Sü. eine zwanzigfache Erhöhung der Permeabilität. Der Zeitpunkt der Untersuchung bei Fall Kr. (4. Tag der Rekonvaleszenz) macht ohne weiteres den niederen Wert verständlich. Auch der Durch tritt der Typhusund Paratyphusagglutinine erfolgt bei Fleckfieber in derselben oder ähnlichen Weise wie der der spezifischen Agglutinine. Damit fällt der eventuelle Einwand, daß der Nachweis von Typhusagglutininen beim Abdominalis deshalb mißlingt, weil diese schwerer in den Liquor übertreten als die Fleckfieberagglutinine. Geringgradige Differenzen, die das eine oder anderemal bestehen, lassen sich in derselben Weise auch gegenüber $\mathrm{X}_{2}$ und $\mathrm{X}_{19}$ feststellen.

Der Schluß, daß bei jedem Falle von Fleckfieber in einem bestimmten Stadium der Erkrankung (um die Zeit der beginnenden Entfieberung) sich eine erhöhte Permeabilität der Meningen findet, scheint uns auf Grund dieser Feststellungen gerechtfertigt. Die Tatsache, daß diese auch bei leichten und leichtesten Erkrankungen bestoht, ist sehr auffallend und beweist, daß eine Alteration der Meningealgefäße ebenso zum Krankheitsbild des Fleckfiebers gehört wie das Exanthem. Die Beobachtungen von Soucek, der unabhängig von allen Autoren stets auf die zerebralen Symptome hingewiesen hat, die er uns in jedem Falle von Fleckfieber demonstrieren konnte, erhalten durch diese experimentellen Befunde eine objektive Stütze.

Von Wichtigkeit wäre die Entscheidung der Frage, ob man angesichts dieser Feststellungen von einer Meningitis sprechen kann, oder ob nur eine Reizung der Meningen (Meningismus) vorliegt. Wir können diesbezüglich nur soviel sagen, daß bei Meningismus die Hämolysinreaktion negativ ist. Die sonstigen Veränderungen im Liquor, die auch von Müller-Deha m als Druckvermehrung, zartes, zellarmes Gerinnsel beschrieben wurden, bieten nicht das Bild, das wir von einer Meningitis zu sehen gewohnt sind, obzwar wir in manchen Fällen eine starke Zellvermehrung (poly- und mononukleäre Leukozyten) und auch Eiweißvermehrung konstatieren konnten. Nur die genaue anatomische Untersuchung der Meningen kann uns darüber Klarheit verschaffen, ob die Veränderungen der Meningen entzündlicher Natur sind, oder ob es sich nur um Störungen ihrer filtrierenden Fähigkeit handelt. 